\title{
Systematic Evaluation of Current Possibilities to Determine Left Ventricular Volumes by Echocardiography in Patients after Myocardial Infarction
}

\author{
Stephan Stoebe ${ }^{1}$, Adrienn Tarr², Tudor Trache ${ }^{1}$, Jens-Gerrit Kluge ${ }^{1}$, Dietrich Pfeiffer ${ }^{1}$, \\ Andreas Hagendorff ${ }^{1}$ \\ ${ }^{1}$ Department of Cardiology-Angiology, University of Leipzig, Leipzig, Germany \\ ${ }^{2}$ Department of Internal Medicine \& Cardiology Center, Faculty of Medicine, University of Szeged, Szeged, Hungary \\ Email: stephan.stoebe@gmx.de
}

Received March 13, 2012; revised April 8, 2012; accepted April 23, 2012

\begin{abstract}
Purpose: The aim of the present study was to evaluate the diagnostic accuracy for quantification of left ventricular (LV) volumes and LV ejection fraction (LVEF) with current echocardiographic methods of planimetry for analysis of LV remodeling after myocardial infarction in daily clinical routine. Methods: 26 patients were investigated directly after interventional therapy at hospital pre-discharge and at 6 months follow-up. Standardized 2D transthoracic native and contrast echocardiography were performed in all patients. Due to methodological aspects the results of LV volumes and LVEF using native echocardiography were compared to the results of LV opacification (LVO) imaging for analysis in mono-, bi- and triplane data sets using the Simpson's rule. In addition corresponding multidimensional data sets were analyzed. Results: The assessment of LV volumes and LVEF is more accurate with contrast echocardiography. The comparison of LV volumes and LVEF shows significant increases using contrast echocardiography $(p<0.001)$. Larger left ventricular end-diastolic volumes (LVEDV) are measured at follow up $(\mathrm{p}<0.05)$. Significant differences $(\mathrm{p}<0.001)$ are found for the determination of LVEDV and LVEF relating to apical mono-, bi-, tri- and multiplane data sets. Standard deviations of the triplane approach, however, are significantly lower than using other modalities. Conclusion: Depending on the localization of the myocardial infarction LV volumes and LVEF are less reliably evaluated using the mono- or biplane approach. According to standardization and simultaneous acquisition of all LV wall segments the triplane approach is currently the best approach to determine LV systolic function. In addition, contrast echocardiography is indicated to improve endocardial border delineation in patients using the triplane or multiplane approach. To our knowledge the present study is the first systematic evaluation of all current possibilities for determination of LV volumes and LVEF by native and contrast echocardiography.
\end{abstract}

Keywords: Contrast Echocardiography; Left Ventricular Systolic Function; Left Ventricular Volumes; Remodeling; Myocardial Infarction; LVO Imaging

\section{Introduction}

Accurate left ventricular (LV) volume determination by echocardiography is still a challenge in clinical practice. Nayyar and coworkers described that the interobserver variability for left ventricular volume determination can be significantly reduced by better endocardial border detection due to contrast echocardiography [1]. However, especially in patients with regional wall motion abnormalities quantification of left ventricular volumes and ejection fraction (EF) strongly depends on the views which were used for planimetry. Thus, the present study focusses on the variances of volumes in patients with regional myocardial infarction determined by performing planimetry in different echocardiographic views. This is important because changes of volumes during drug treatment after myocardial infarction can only be detected if determination of volumes are acceptable accurate and reproducible.

After acute myocardial infarction (AMI) the heart undergoes a process of structural and functional changes [2, 3]. This process is described as "remodeling" characterized by left ventricular dilatation, eccentric hypertrophy, wall thinning in the non-infarcted area and LV spherizisation as well as aneurysm formation in the infarcted area $[4,5]$.

Echocardiography is accepted for quantification of LV volumes and LVEF as well as for determination of regional wall motion abnormalities in patients after AMI. 
The determination of left ventricular end-diastolic volumes (LVEDV), left ventricular end-systolic volumes (LVESV) and LVEF has individual prognostic value and is used to evaluate $\mathrm{LV}$ remodeling at follow-up investigations $[3,6,7]$. For improved diagnostics and especially in case of bad acoustic windows contrast echocardiography is established [8]. With respect to different infarction patterns the determination and analysis of LVEF and LV volumes should be analyzed by different echocardiographic modalities for early detection of remodeling.

The aim of the present study was a systematic evaluation of all current possibilities for determination of $\mathrm{LV}$ volumes and LVEF by native and contrast echocardiography. Thus, the mono-, bi-, tri- and multiplane approach was used for planimetry in patients after AMI despite the fact that regional wall motion abnormalities will have a significant impact on LV volumes. Furthermore, potential changes of the volume parameters due to an early remodeling process after AMI were evaluated. All patients were treated with valsartan or ACE (= angiotensin-converting enzyme) inhibitors.

\section{Methods}

In the present prospective study 57 patients were enrolled from June 2009 to March 2011. Ultimately 46 of 57 patients were enclosed and 37 underwent the study completely. The analysis focuses, however, only on patients with AMI. Thus, a total of 26 patients (age $63 \pm 13$ ) after AMI were included. The study population consists of 8 female and 18 male patients. All patients were treated with valsartan or ACE inhibitor in combination with beta blockers, respectively. Percutaneous coronary angioplasty was performed in a time interval of 1 to 6 hours after onset of symptoms. All patients were treated with aspirin, heparin, nitrates and beta blockers after onset of symptoms and prior to the intervention. Echocardiography was performed directly after interventional therapy at hospital pre-discharge and at follow-up 6 months later.

Inferior AMI was documented in 6 patients and anterior AMI was documented in 20 patients. Standardized transthoracic echocardiography as well as contrast echocardiography was performed in all patients using a GE Vivid 7 system (GE Healthcare) with a M4S phased array and a $3 \mathrm{~V}$ probe [9]. LVEDV, LVESV and LVEF were determined using the modified Simpson's rule in mono-, bi-, tri- and multiplane data sets. LV volumes and LVEF were obtained by endocardial border delineation at end-diastole and end-systole. With the method of disc left ventricular volumes result from a summation of elliptical discs perpendicular to the longitudinal axis of the left ventricle [10]. Monoplane analysis was obtained from the apical long axis view whereas biplane analysis was obtained using the apical 2- and 4-chamber view. The triplane analysis enables a simultaneous acquisition of all three standardized apical views. Real-Time-3D-Echocardiography (RT3DE) permits three-dimensional visualization of cardiac structures by acquisition of 3D data sets detecting the entire heart in a $90^{\circ} \times 90^{\circ}$ sector. The $\mathrm{LV}$ systolic function was evaluated by automated endocardial border detection. For the quantification of multiplane data sets automated volume analysis (GE, 4D AutoLVQ software) was used [10,11]. Contrast administration was performed by repetitive bolus injections of $0.3-0.4 \mathrm{ml}$ of SonoVue (BRACCO). The data sets were analyzed offline using the EchoPAC software version 110.1.1. In 10 of 26 randomly selected patients LVEDV, LVESV and LVEF was determined by another experienced investigator. The interobserver variability was assessed for each method using native and contrast echocardiography. Both investigators used the same data sets and were blinded to each other's results.

\section{Statistics}

The statistical analysis was obtained by SPSS Statistics software version 17.0. Mean and standard deviations (SD) were calculated for LVEDV, LVESV and LVEF determined by native and contrast echocardiography using the different modalities. Both data of the parameters determined by native and contrast echocardiography and mono-, bi-, tri- and multiplane approaches were compared to each other using paired t-Test.

\section{Results}

The assessment of LVEDV, LVESV and LVEF is more accurate using contrast echocardiography. With respect to standardization and adequate image quality LVEDV, LVESV and LVEF show significant increases using contrast echocardiography in comparison to native echocardiography (Figures 1 and 2, Table 1 and Figures 3-8). In addition, due to reduced image quality in native echocardiography (defined by insufficient endocardial border detection in more than 2 segments) 5 patients could not be analyzed without contrast, whereas endocardial contour detection was possible in all patients using contrast. Results of LVEDV, LVESV and LVEF measurements were compared by paired t-Test $(\mathrm{p}<0.001)$. Variances of LVEDV and LVEF are lower in contrast echocardiography compared to native echocardiography, respectively (Table 1). Although in some cases LVEDV was reduced in comparison to the investigation at hospital pre-discharge mean LVEDV was increased at the follow up after 6 months ( $<<0.05$, Figures 3-8).

In detail, this mean increase of LVEDV with a distinct increase of more than $10 \mathrm{ml}$ within the follow up period was assessed in 10 patients. In this cohort in 6 patients the infarcted area was larger than $40 \%$ of the myocardium, 


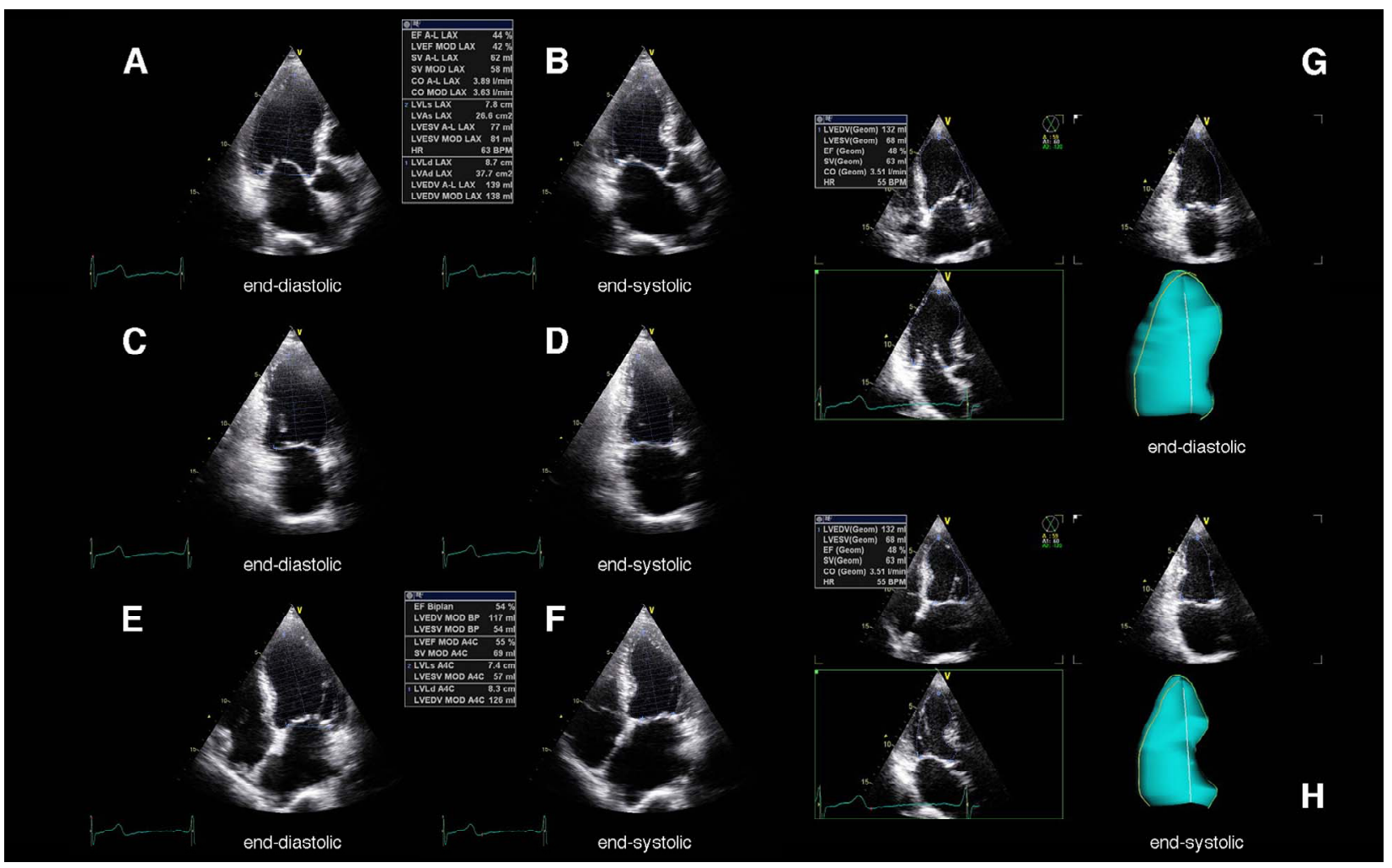

Figure 1. Determination of left ventricular systolic function by the apical mono-, bi- and triplane approach using native echocardiography in a patient with septal myocardial infarction. Above the apical long axis view (A), (B), the apical 2-chamber view (C), (D) and the apical 4-chamber view (E), (F) are shown at end-diastole (A), (C), (E) and end-systole (B), (D), (F). On the right the simultaneous visualization of all apical views obtained by the triplane approach including a corresponding dynamic $L V$ cast is shown at end-diastole $(G)$ and end-systole $(H)$.

Table 1. Mean \pm standard deviation and p-values (paired t-Test) of LVEDV, LVESV and LVEF obtained by the apical mono-, bi-, tri- and multiplane approach using native and contrast echocardiography. Both the investigation at hospital pre-discharge (1) and the follow-up (2) are shown.

\begin{tabular}{|c|c|c|c|c|c|c|c|c|c|}
\hline $\begin{array}{c}n=21 \text { (native) } \\
n=26 \text { (contrast) }\end{array}$ & $\begin{array}{l}\text { LVEDV } \\
\text { native }\end{array}$ & $\begin{array}{l}\text { LVEDV } \\
\text { contrast }\end{array}$ & p-values & $\begin{array}{c}\text { LVESV } \\
\text { native }\end{array}$ & $\begin{array}{l}\text { LVESV } \\
\text { contrast }\end{array}$ & p-values & $\begin{array}{l}\text { LVEF } \\
\text { native }\end{array}$ & $\begin{array}{l}\text { LVEF } \\
\text { contrast }\end{array}$ & p-values \\
\hline Monoplane (1) & $126 \pm 21$ & $143 \pm 19$ & $<0.001$ & $74 \pm 15$ & $78 \pm 14$ & $<0.001$ & $41 \pm 8$ & $46 \pm 6$ & $<0.001$ \\
\hline Biplane (1) & $110 \pm 17$ & $127 \pm 16$ & $<0.001$ & $58 \pm 12$ & $62 \pm 11$ & $<0.001$ & $48 \pm 7$ & $51 \pm 6$ & $<0.001$ \\
\hline Triplane (1) & $119 \pm 16$ & $135 \pm 14$ & $<0.001$ & $64 \pm 10$ & $69 \pm 9$ & $<0.001$ & $46 \pm 5$ & $49 \pm 4$ & $<0.001$ \\
\hline Multiplane (1) & $121 \pm 18$ & --- & & $66 \pm 13$ & --- & & $45 \pm 7$ & --- & \\
\hline $\begin{array}{c}n=21 \text { (native) } \\
n=26 \text { (contrast) }\end{array}$ & $\begin{array}{l}\text { LVEDV } \\
\text { Native }\end{array}$ & $\begin{array}{l}\text { LVEDV } \\
\text { contrast }\end{array}$ & p-values & $\begin{array}{l}\text { LVESV } \\
\text { native }\end{array}$ & $\begin{array}{l}\text { LVESV } \\
\text { contrast }\end{array}$ & p-values & $\begin{array}{l}\text { LVEF } \\
\text { native }\end{array}$ & $\begin{array}{c}\text { LVEF } \\
\text { contrast }\end{array}$ & p-values \\
\hline Monoplane (2) & $135 \pm 19$ & $152 \pm 17$ & $<0.001$ & $77 \pm 16$ & $79 \pm 15$ & $<0.001$ & $44 \pm 7$ & $48 \pm 6$ & $<0.001$ \\
\hline Biplane (2) & $117 \pm 18$ & $133 \pm 17$ & $<0.001$ & $59 \pm 12$ & $63 \pm 10$ & $<0.001$ & $49 \pm 7$ & $52 \pm 6$ & $<0.001$ \\
\hline Multiplane (2) & $130 \pm 18$ & --- & & $69 \pm 12$ & --- & & $47 \pm 7$ & --- & \\
\hline
\end{tabular}

and in 4 patients no reflow was observed after treatment of the culprit artery. In 5 patients LVEDV decreased, in 11 patients LVEDV did not significantly change. Sig- nificant differences of LVEDV and LVEF ( $<<0.001$ ) were also found using the apical mono-, bi-, triplane approach (Table 1, Figures 1 and 2) as well as the multi- 


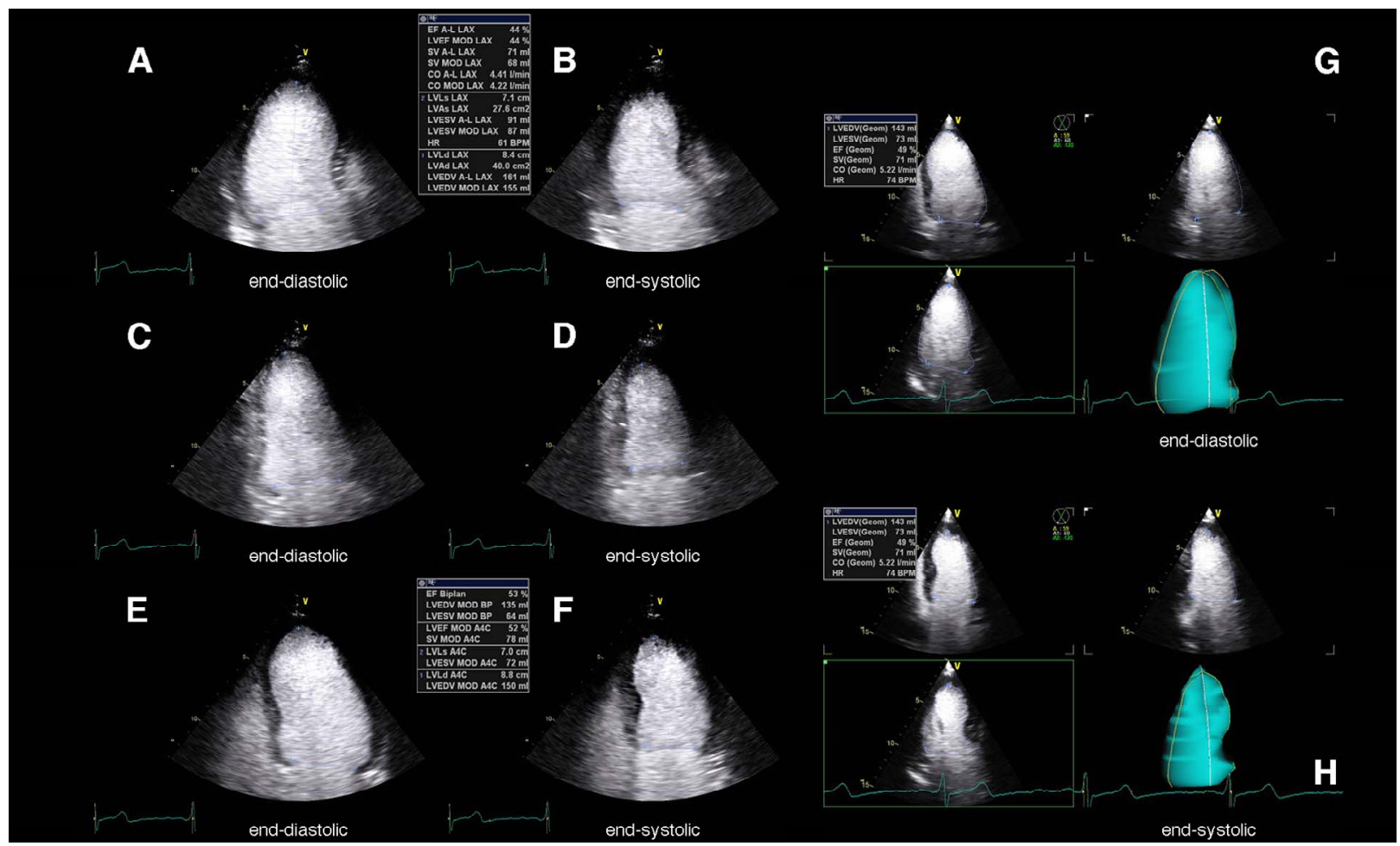

Figure 2. Determination of left ventricular systolic function by the apical mono-, bi- and triplane approach using contrast echocardiography in a patient with septal myocardial infarction. Above the apical long axis view (A), (B), the apical 2-chamber view (C), (D) and the apical 4-chamber view (E), (F) are shown at end-diastole (A), (C), (E) and end-systole (B), (D), (F). On the right the simultaneous visualization of all apical views obtained by the triplane approach including a corresponding dynamic LV cast is shown at end-diastole $(G)$ and end-systole $(H)$.

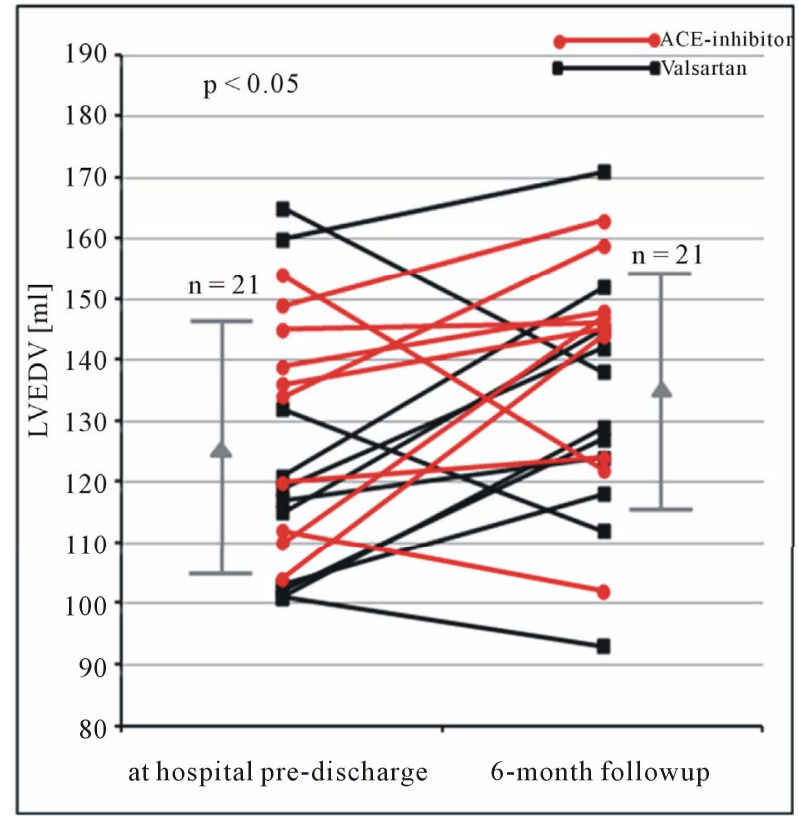

Figure 3. LVEDV (ml) obtained by the monoplane approach using native echocardiography at hospital pre-discharge and 6 months follow-up in patients after AMI. Changes of LVEDV are shown for each patient. In addition mean and standard deviation are given (LVEDV = left ventricular end-diastolic volume).

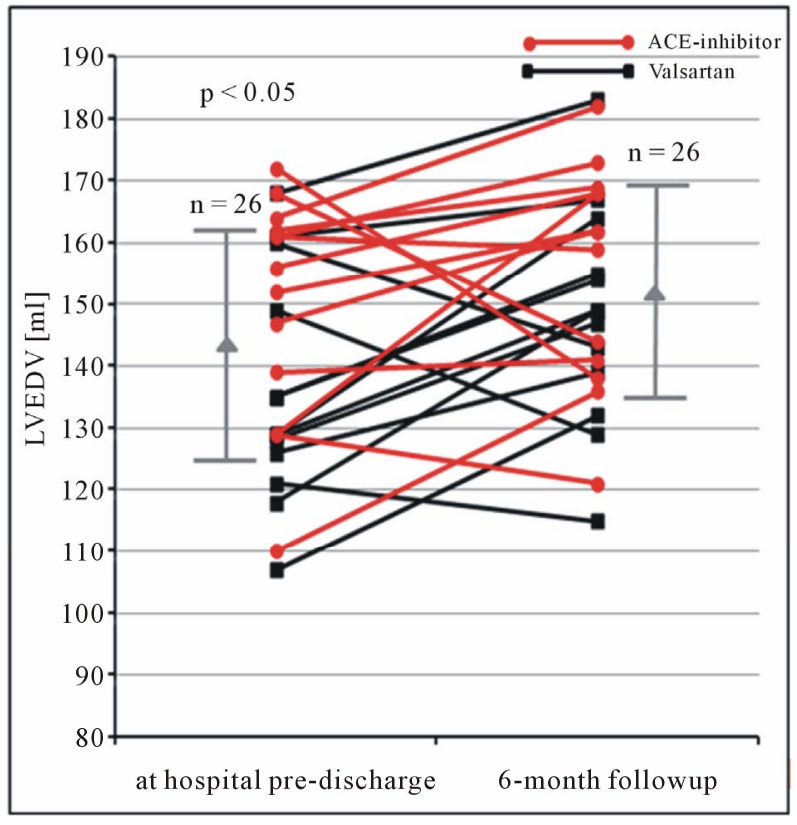

Figure 4. LVEDV (ml) obtained by the monoplane approach using contrast echocardiography at hospital predischarge and 6 months follow-up in patients after AMI. Changes of LVEDV are shown for each patient. In addition mean and standard deviation are given (LVEDV = left ventricular end-diastolic volume). 


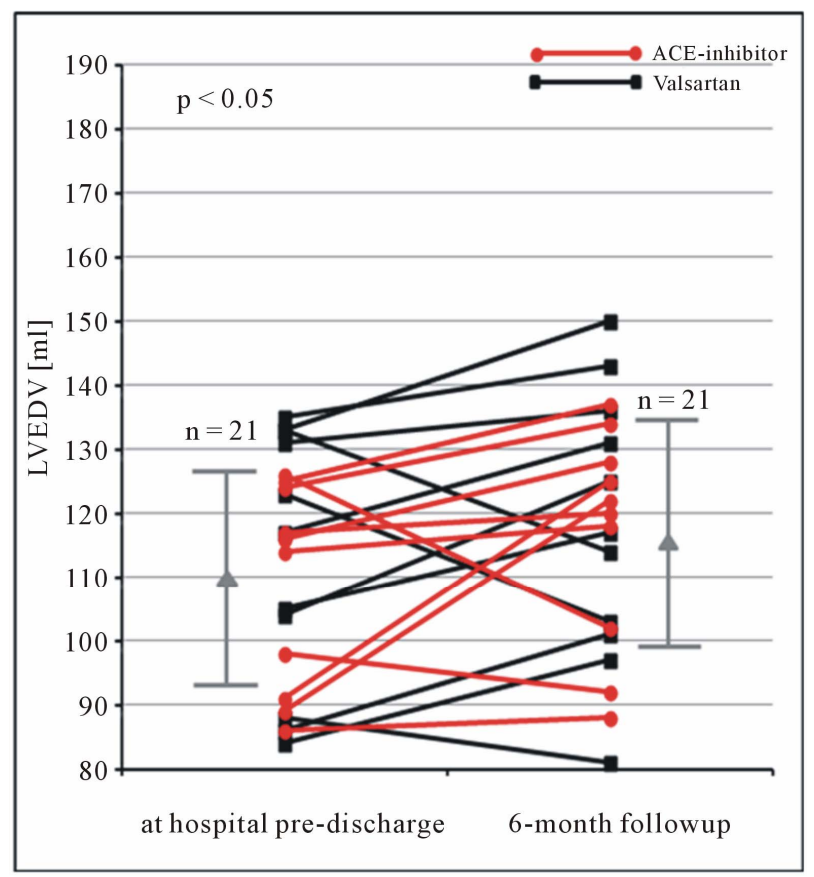

Figure 5. LVEDV (ml) obtained by the biplane approach using native echocardiography at hospital pre-discharge and 6 months follow-up in patients after AMI. Changes of LVEDV are shown for each patient. In addition mean and standard deviation are given $(\mathrm{LVEDV}=$ left ventricular end-diastolic volume).

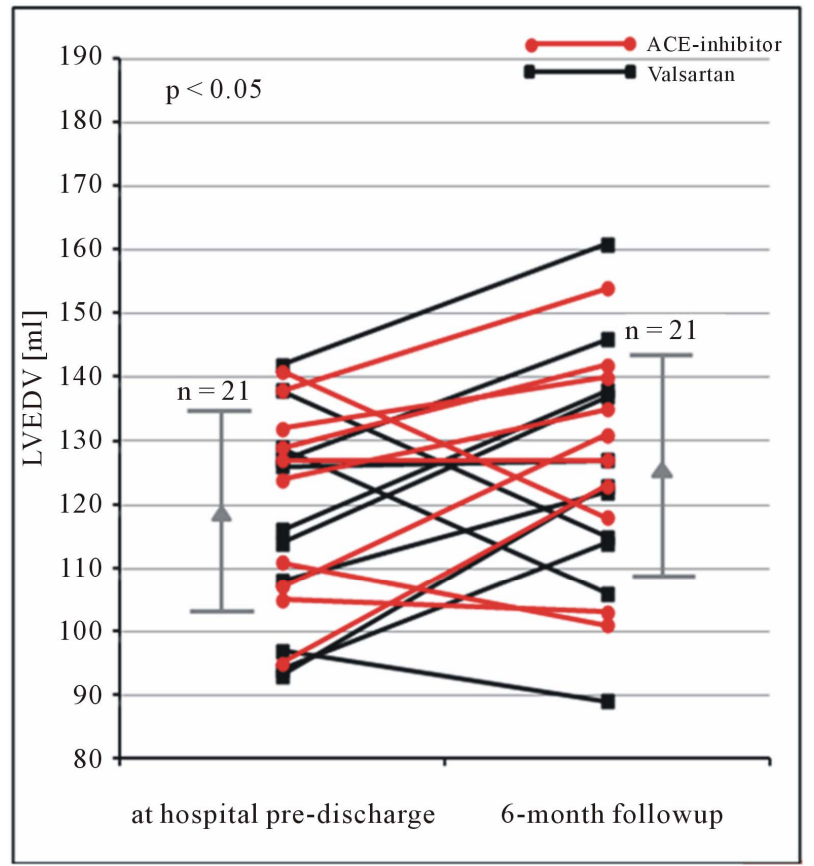

Figure 7. LVEDV (ml) obtained by the triplane approach using native echocardiography at hospital pre-discharge and 6 months follow-up in patients after AMI. Changes of LVEDV are shown for each patient. In addition mean and standard deviation are given (LVEDV = left ventricular end-diastolic volume).

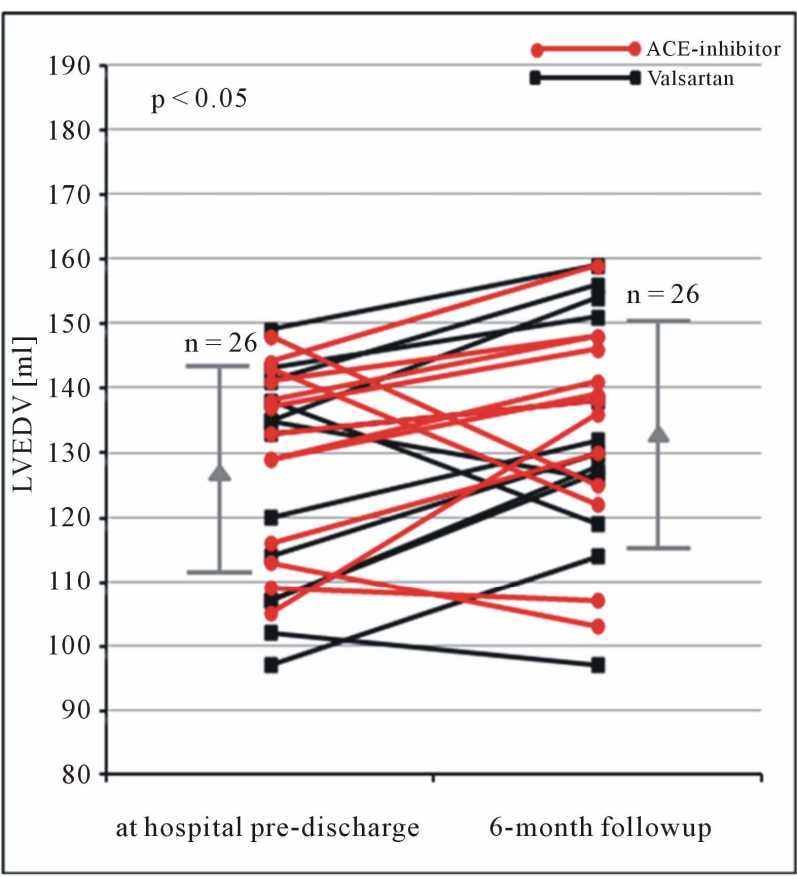

Figure 6. LVEDV (ml) obtained by the biplane approach using contrast echocardiography at hospital pre-discharge and 6 months follow-up in patients after AMI. Changes of LVEDV are shown for each patient. In addition mean and standard deviation are given (LVEDV = left ventricular end-diastolic volume).

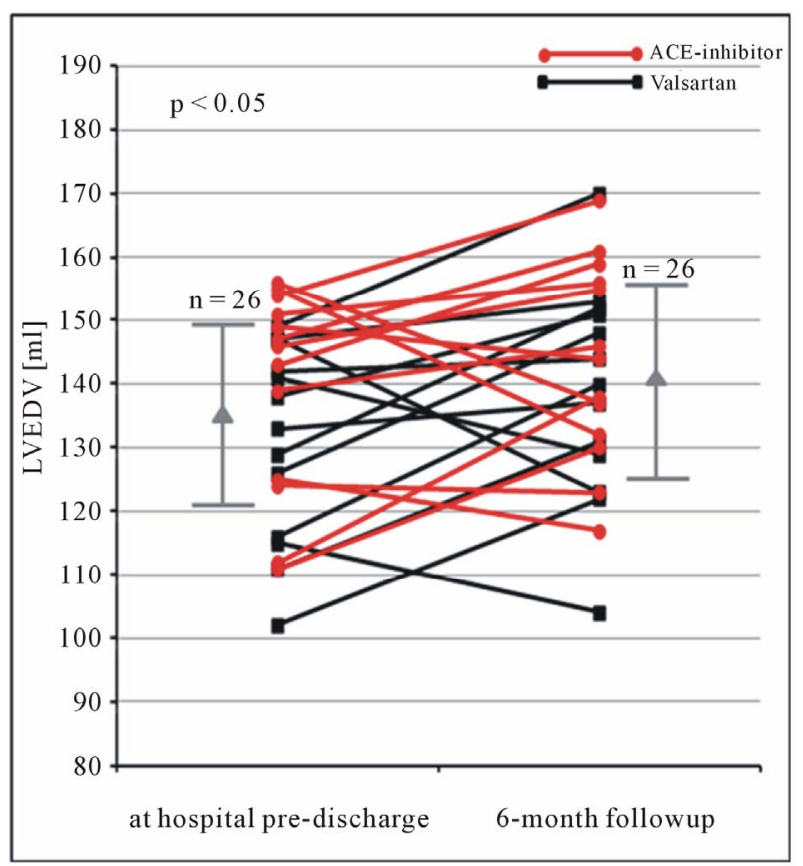

Figure 8. LVEDV (ml) obtained by the triplane approach using contrast echocardiography at hospital pre-discharge and 6 months follow-up in patients after AMI. Changes of LVEDV are shown for each patient. In addition mean and standard deviation are given (LVEDV = left ventricular end-diastolic volume). 
dimensional approach (Figure 9). Standard deviations of the triplane approach, however, were significantly lower than using the mono-, bi- and multidimensional approach (Table 1). In most of the contrast applications multidimensional imaging was possible ( $\mathrm{n}=22$ patients/26 patients, Figure 10). Measurements for LVEDV, LVESV and LVEF determined by another investigator were only slightly different. Mean differences between both investigators were $\pm 7 \%$ for LVEDV, $\pm 5 \%$ for LVESV and $\pm 6 \%$ for LVEF using native and contrast echocardiography.

\section{Discussion}

To our knowledge the present study is the first systematic evaluation of all current possibilities of planimetry for determination of LV volumes and LVEF by native and contrast echocardiography.

The main findings of the present study are:
1) Endocardial contour detection was feasible in all patients using contrast whereas not all patients could be analyzed with native echocardiography;

2) The assessment of changes of LV volumes and LVEF is more accurate with contrast echocardiography;

3) In comparison to native echocardiography larger left ventricular end-diastolic volumes are determined by contrast echocardiography;

4) Significant differences are found for LVEDV and LVEF due to the mono-, bi-, tri- and multiplane approach;

5) Standard deviations of the triplane approach are lower than using other modalities.

The accuracy of echocardiographic measurements is often criticized because of problems in reproducibility and interobserver variability [11,12]. Despite of these criticisms echocardiography is still the most used method

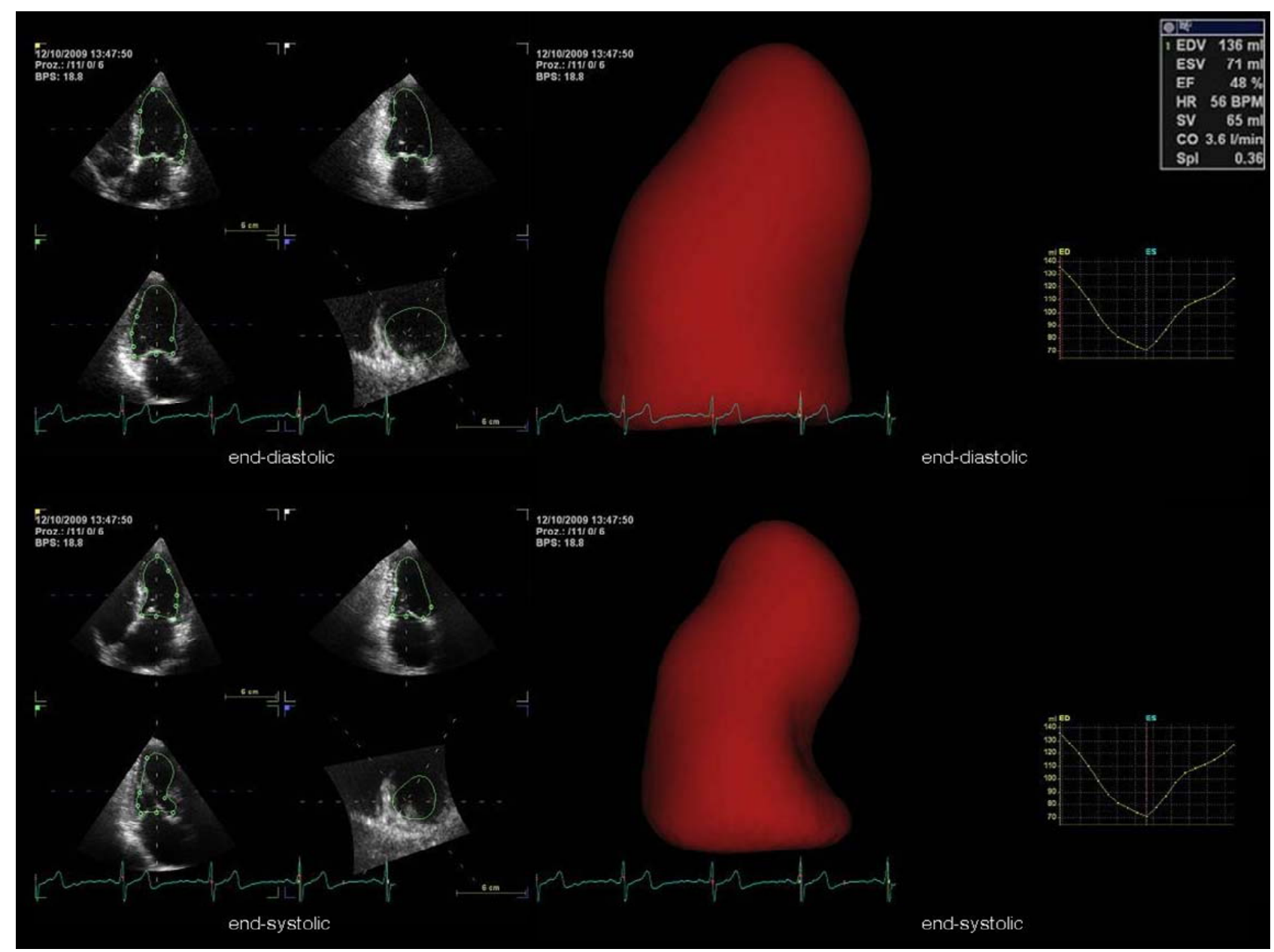

Figure 9. Determination of left ventricular systolic function by multiplane data sets with semi-automated endocardial border detection (4D AutoLVQ software). On the left the simultaneous visualization of the apical long axis view, the apical 2-chamber view and the apical 4-chamber view is shown at end-diastole and end-systole. In addition, a 3D LV volume reconstruction in terms of a surface-rendered LV cast and a corresponding volume-time plot are shown at end-diastole and end-systole. 


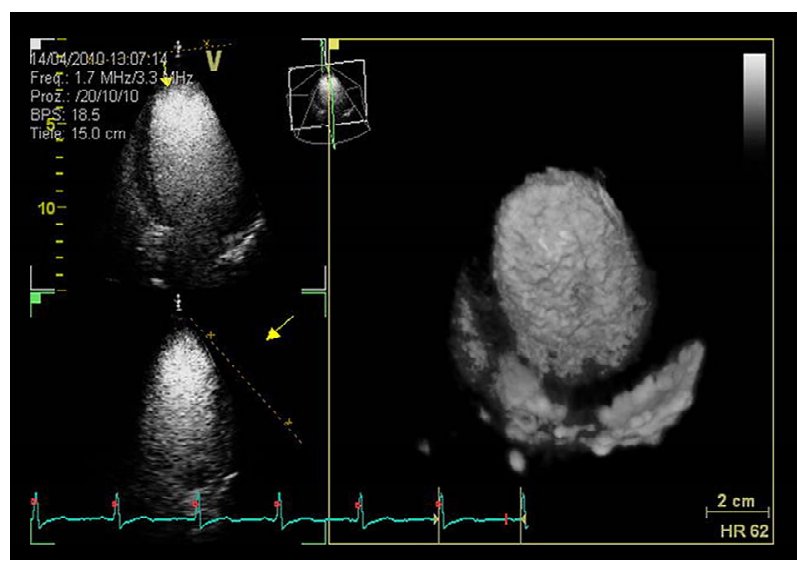

Figure 10. Visualization of left ventricular systolic function by opacification of the cardiac cavities in patients after myocardial infarction using the multiplane approach with contrast echocardiography.

in cardiac imaging. In echocardiography standardization is necessary to improve the accuracy of diagnostic reproducibility, interobserver variability and comparability of findings especially in follow-up investigations. In the standardized apical long axis view the anteroseptal and the posterior left ventricular wall is visualized [9]. In case of strict anteroseptal or posterior myocardial infarction this leads to underestimation of the global left ventricular systolic function using the apical monoplane approach. Otherwise the global left ventricular systolic function will be overestimated by the biplane approach (Figures 1 and 2). The modified Simpson's rule represents the currently recommended method of choice for quantification of left ventricular systolic function using the apical 2- and 4-chamber view [4,13]. Inferior, anterior, inferoseptal and lateral section of the left ventricular wall will be detected whereas wall abnormalities in the anteroseptal or posterior section will not be detected. For this reason LVEDV, LVESV and LVEF differ depending on the localization of the myocardial infarction and the method (mono-, bi-, or triplane approach) is used. Underestimations as well as overestimations of the global left ventricular systolic function can be obtained by LV foreshortening and not standardized views. The triplane approach enables the standardization of all apical views simultaneously, which has a significant impact on better standardization of the apical views. Thus, the present data show that the triplane approach with contrast echocardiography can be seen as the best method for quantification of LV systolic function.

Contrast echocardiography is indicated due to better standardization and improvement of endocardial border delineation to improve the analysis of LV systolic function especially in patients with inadequate image quality in more than two segments [8]. Contrast echocardiography, however, opacifies the spaces between the trabecu- lations of the left ventricular wall causing larger left ventricular end-diastolic volumes by tracing the endocardial border in comparison to native echocardiography [13]. Thus, it can be explained that the assessment by contrast echocardiography leads to higher values of $\mathrm{LV}$ volumes and LV ejection fraction.

Our data documents the necessity of contrast echocardiography for accurate determination of $\mathrm{LV}$ volumes and LV ejection fraction in patients after AMI in the daily clinical routine, if distinct volume changes will be determined according to the data of Nayyar et al. [1].

The determination of left ventricular systolic function by multiplane data sets via semi-automated border detection (Figure 9) is currently not established in clinical routine. Although image quality, frame rate and temporal and spatial resolution in real-time-3D-echocardiography (RT3DE) are lower than in 2D echocardiography it is verified that RT3DE has shown to be more accurate and reproducible for the determination of left ventricular volumes and left ventricular systolic function [11,14-16]. In RT3DE left ventricular volumes are directly calculated by a mathematical model that enables a uniform tracing of the whole endocardial surface $[11,15]$. Due to standard deviations of LVEDV, LVESV and LVEF resulting from multiplane data sets its determination seems to be at least as reliable as measurements obtained by the biplane approach. Thus, in case of good image quality RT3DE is more accurate than conventional 2D echocardiography. Improvements have to be made referring to faster acquisition and application of the analyzed data sets. Currently automatic border detection can be seen as an important additional method for quantification of left ventricular function in clinical routine and will probably gain more importance in the future.

\section{Limitations}

The present study focuses on methodological aspects. Despite of the relatively small number of patients the statistical analysis proves the significant improvements of image quality and thus more accurate determination of LV volumes and LVEF with contrast echocardiography. The small cohort of patients does not allow conclusion about LV volume changes due to different drug trerapy.

\section{Conclusion}

Depending on the localization of the myocardial infarction LV volumes and LVEF are less accurate to be evaluated using the mono- or biplane approach in comparison to the triplane approach. Thus, with respect to standardization the simultaneous acquisition of all left ventricular wall segments with the triplane approach is currently the best approach to determine LV ejection fraction as a conventional parameter for LV systolic 
function. In addition, contrast echocardiography is indicated to improve endocardial border delineation in patients using the triplane and multiplane approach for better accuracy. With respect to improvements in echocardiography the evaluation of LV volumes in multiplane data sets with contrast echocardiography will enter the diagnostic scenario soon.

\section{Acknowledgements}

The present study was supported by Novartis (CVAL489BDE29T/CSR).

\section{REFERENCES}

[1] S. Nayyar, A. Magalski, T. M. Khumri, M. Idupulapati, C. N. Stoner, L. L. Kusnetzky, T. R. Coggins, B. A. Morris and M. L. Main, "Contrast Administration Reduces Interobserver Variability in Determination of Left Ventricular Ejection Fraction in Patients with Left Ventricular Dysfunction and Good Baseline Endocardial Border Delineation,” The American Journal of Cardiology, Vol. 98, No. 8, 2006, pp. 1110-1114. doi:10.1016/j.amjcard.2006.05.038

[2] K. Thygesen, J. S. Alpert and H. D. White, "Universal Definition of Myocardial Infarction,” European Heart Journal, Vol. 28, No. 20, 2007, pp. 2525-2538. doi: 10.1093/eurheartj/ehm355

[3] The Joint European Society of Cardiology/American College of Cardiology Committee, "Myocardial Infarction Redefined-A Consensus Document of The Joint European Society of Cardiology/American College of Cardiology Committee for the Redefinition of Myocardial Infarction,” European Heart Journal, Vol. 21, No. 18, 2000, pp. 1502-1513. doi:10.1053/euhj.2000.2305

[4] R. M. Lang, M. Bierig, R. B. Devereux, F. A. Flachskampf, E. Foster, P. A. Pellikka, M. H. Picard, M. J. Roman, J. Seward, J. Shanewise, S. Solomon, K. T. Spencer, M. St. J. Sutton and W. Stewart, "Recommendations for Chamber Quantification,” European Journal of Echocardiography, Vol. 7, No. 2, 2006, pp. 79-108. doi:10.1016/j.euje.2005.12.014

[5] J. J. Gajarsa and R. A. Kloner, "Left Ventricular Remodeling in the Post-Infarction Heart: A Review of Cellular, Molecular Mechanisms, and Therapeutic Modalities,” Heart Failure Reviews, Vol. 16, No. 1, 2011, pp. 13-21. doi:10.1007/s10741-010-9181-7

[6] M. Wong, L. Staszewsky, R. Latini, S. Barlera, R. Glazer, N. Aknay, A. Hester, I. Anand and J. N. Cohn, "Severity of Left Ventricular Remodeling Defines Outcomes and Response to Therapy in Heart Failure: Valsartan Heart Failure Trial (Val-HeFT) Echocardiographic Data,” Journal of the American College Cardiology, Vol. 43, No. 11, 2004, pp. 2022-2027. doi:10.1016/j.jacc.2003.12.053

[7] N. P. Nikolay, C. Constantin, P. H. Lo, J. Ghosh, E. I. Lu- kaschuk, A. Bennett, S. Hurren, F. Alamgir, A. L. Clark and J. G. F. Cleland, "New Generation 3-Dimensional Echocardiography for Left Ventricular Volumetric and Functional Measurements: Comparison with Cardiac Magnetic Resonance,” European Journal of Echocardiography, Vol. 7, No. 5, 2006, pp. 365-372. doi:10.1016/j.euje.2005.09.005

[8] L. Agati, G. Tonti, L. Galiuto, V. D. Bello, S. Funaro, M. P. Madonna, B. Garramone and F. Magri, "Quantification Methods in Contrast Echocardiography,” European Journal of Echocardiography, Vol. 6, Suppl. 2, 2005, pp. S14S20. doi: 10.1016/S1525-2167(05)80724-3

[9] A. Hagendorff, "Transthoracic Echocardiography in Adult Patients-A Proposal for Documenting a Standardized Investigation,” Ultraschall in der Medizin, Vol. 29, No. 4, 2008, pp. 344-374. doi: 10.1055/s-2008-1027491

[10] D. Muraru, L. P. Badano, G. Piccoli, P. Gianfagna, L. del Mestre, D. Ermacora and A. Proclemer, "Validation of a Novel Automated Border-Detection Algorithm for Rapid and Accurate Quantitation of Left Ventricular Volumes Based on Three-Dimensional Echocardiography," European Journal of Echocardiography, Vol. 11, No. 4, 2010, pp. 359-368. doi:10.1093/ejechocard/jep217

[11] M. J. Monaghan, "Role of Real Time 3D Echocardiography in Evaluating the Left Ventricle,” Heart, Vol. 92, No. 1, 2006, pp. 131-136. doi:10.1136/hrt.2004.058388

[12] R. Hoffmann, S. von Bardeleben, F. ten Cate, A. C. Borges, J. Kasprzak, C. Firschke, S. Lafitte, N. Al-Saadi, S. Kuntz-Hehner, M. Engelhardt, H. Becher and J. L. Vanoverschelde, “Assessment of Systolic Left Ventricular Function: A Multi-Centre Comparison of Cineventriculography, Cardiac Magnetic Resonance Imaging, Unenhanced and Contrast-Enhanced Echocardiography," European Heart Journal, Vol. 26, No. 6, 2005, pp. 607-616. doi:10.1093/eurheartj/ehi083

[13] R. Olszewski, J. Timperley, S. Cezary, M. Monaghan, P. Nihoyannopoulis, R. Senior and H. Becher, "The Clinical Applications of Contrast Echocardiography,” European Journal of Echocardiography, Vol. 8, No. 3, 2007, pp. S13-S23. doi:10.1016/j.euje.2007.03.021

[14] K. Y. E. Leung and J. G. Bosch, “Automated Border Detection in Three-Dimensional Echocardiography: Principles and Promises," European Journal of Echocardiography, Vol. 11, No. 2, 2010, pp. 97-108. doi:10.1093/ejechocard/jeq005

[15] H. F. J. Mannaerts, J. A. van der Heide, O. Kamp, M. G. Stoel, J. Twisk and C. A. Visser, "Early Identification of Left Ventricular Remodeling after Myocardial Infarction, Assessed by Transthoracic 3D Echocardiography,” European Heart Journal, Vol. 25, No. 8, 2004, pp. 680-687. doi:10.1016/j.ehj.2004.02.030

[16] V. Mor-Avi, L. Sugeng and R. M. Lang, "Real-Time 3-Dimensional Echocardiography: An Integral Component of the Routine Echocardiographic Examination in Adult Patients?” Circulation, Vol. 119, 2009, pp. 314-329. doi:10.1161/CIRCULATIONAHA.107.751354 